\title{
Deseo intenso por alimentos apetecibles: validación y estandarización del Food Craving Questionnaire-Trait en México
}

\author{
María Delfina Marín-Soto, ${ }^{1}$ Cruz Vargas-De León, ${ }^{1}$ Nadia Mabel Pérez-Vielma, ${ }^{2}$ \\ Margarita Castillo-Ramírez, ${ }^{3}$ Ángel Miliar-García, ${ }^{2}$ María Magdalena Murillo-Tovar, ${ }^{2}$ \\ Bárbara Méndez-Peña ${ }^{2}$ y Víctor Ricardo Aguilera-Sosa ${ }^{2 *}$ \\ ${ }^{1}$ Instituto Politécnico Nacional, Escuela Superior de Medicina; ${ }^{2}$ Instituto Politécnico Nacional, Centro Interdisciplinario de Ciencias de la Salud \\ Unidad Santo Tomás; ${ }^{3}$ Secretaría de Salud de la Ciudad de México. Ciudad de México, México
}

\section{Resumen}

Introducción: El food craving o "ansia por comer" es una respuesta motivacional y fisiológica por comer alimentos específicos, principalmente con alto contenido calórico. Para evaluarlo se usa, entre otros, el Food Craving Questionnaire Trait, estructurado multidimensionalmente y validado en diversos países, el cual ha mostrado ser sensible y adaptable a los cambios contextuales-culturales. Objetivos: Validar y estandarizar el Food Craving Questionnaire-Trait en adultos de la Ciudad de México. Método: Estudio no experimental, transversal y aleatorizado de 1059 sujetos de uno y otro sexo, entre 18 y 84 años; $71.86 \%$ del sexo femenino. Se examinaron propiedades psicométricas con análisis factoriales exploratorios y confirmatorios. Resultados: Se redujeron los factores del cuestionario y los ítems se reorganizaron de forma diferente al original. El análisis factorial confirmatorio mostró ajuste adecuado y estandarización aceptable de los factores. Se encontró alta consistencia interna para el cuestionario global $(\alpha=0.973$ y rho $=0.975)$ para cada uno de los factores. Conclusión: Este estudio determina la viabilidad del Food Craving Questionnaire para población de la Ciudad de México.

PALABRAS CLAVE: Ansia por comer. Conducta alimentaria. Food Craving Questionnaire-Trait.

\section{Intense craving for appetizing foods: validation and standardization of the Food Cravings Questionnaire-Trait in Mexico}

\begin{abstract}
Introduction: Food craving is a motivational and physiological response for eating specific foods, mainly with high caloric content. To assess food craving, self-reports, inventories and questionnaires are used. The Food Cravings Questionnaire-Trait is multi-dimensionally structured and has been validated in several countries, since it is sensitive and adaptable to contextualcultural changes. Objectives: To validate and standardize the Food Cravings Questionnaire-Trait in adults of Mexico City. Method: Non-experimental, cross-sectional, randomized study of 1059 subjects of both genders, between 18 and 84 years of age; $71.86 \%$ of the female gender. Psychometric properties were examined with exploratory and confirmatory factor analyses. Results: The domains of the questionnaire were reduced and the items were reorganized differently from the original version. The confirmatory factor analysis showed an adequate fit and acceptable standardization of factors. High internal consistency was found for the global questionnaire $(\alpha=0.973$ and rho $=0.975)$ for each one of the domains. Conclusion: This study determines the viability of the Food Cravings Questionnaire for the population of Mexico City.
\end{abstract}

KEY WORDS: Food Craving. Alimentary behavior. Food Cravings Questionnaire-Trait.

Correspondencia:

*Víctor Ricardo Aguilera-Sosa

E-mail: psicologiaonline117@gmail.com

CC BY-NC-ND (http://creativecommons.org/licenses/by-nc-nd/4.0/)
Fecha de recepción: 21-06-2019

Fecha de aceptación: 01-07-2019

DOI: 10.24875/GMM.19005358
Gac Med Mex. 2020;156:27-33

Disponible en PubMed

www.gacetamedicademexico.com 


\section{Introducción}

La presencia de alimentos, el ambiente, ${ }^{1}$ la cultura y el aprendizaje están relacionados con la decisión de qué comerá el sujeto; ${ }^{2-4}$ el deseo, la elección, cantidad y frecuencia están relacionados con la incapacidad de inhibir la conducta de ingesta. Se ha hipotetizado que se relaciona con sobreingesta de alimentos con alto contenido calórico, comportamiento similar al de sujetos adictos. ${ }^{5}$

El food craving (FC) es una respuesta fisiológica y comportamental $^{6}$ que desencadena deseo intenso, muy difícil de resistir, ${ }^{7}$ de ingerir alimentos específi$\cos ^{8}{ }^{8}$ particularmente con alto contenido calórico, Por su cercanía con la adicción, el FC está descrito como un factor comórbido de trastornos del comportamiento alimentario, sobrepeso y obesidad. ${ }^{6,9,10}$

Cepeda Benito, Gleaves, Williams y Erath ${ }^{11}$ desarrollaron el Food Craving Questionnaire Trait (FCQ-T), cuestionario multifactorial que engloba componentes conductuales, cognitivos, emocionales y fisiológicos, con $\alpha=0.97$, compuesto por nueve factores:

- Intención y planificación para consumir alimentos (An intention and planning to consume food).

- Anticipación de refuerzo positivo que puede resultar de comer (Anticipation of positive reinforcement that may result from eating).

- Anticipación del alivio de los estados y sentimientos negativos como resultado de comer (Anticipation of relief from negative states and feelings as a result of eating).

- Posible falta de control sobre comer si se come alimentos (Possible lack of control over eating if food is eaten).

- Pensamientos o preocupaciones con la comida (Thoughts or preoccupation with food).

- Antojo como estado fisiológico (Craving as a physiological state).

- Emociones que pueden experimentarse antes o durante los antojos o la comida (Emotions that may be experienced before or during food cravings or eating).

- Señales ambientales que pueden provocar antojos de comida (Environmental cues that may trigger food cravings).

- La culpa que se puede experimentar como resultado de los antojos y/o ceder a ellos (Guilt that may be experienced as a result of cravings and/ or giving into them).

Consta de 37 ítems, en los que se muestra la frecuencia con la que se presenta el "antojo", determinada a partir de una escala Likert: nunca (1), raramente (2), algunas veces (3), a menudo (4), casi siempre (5) y siempre (6).

Avitia et al. ${ }^{12}$ utilizaron la versión original del FCQ-T y Food Craving-State para medirlo en población mexicana, sin embargo, no fueron validados, además de no explicar el procedimiento de aplicación. Por lo tanto, los resultados de esa investigación son cuestionables.

Por la prevalencia combinada de sobrepeso y obesidad en México y dado que el FC es un componente importante de la adicción a la comida azucarada y grasa, es imperativo evaluarlo en población mexicana. El objetivo del presente trabajo fue validar y estandarizar el FCQ-T en adultos de la Ciudad de México.

\section{Método}

Estudio no experimental, transversal y aleatorizado de 1059 adultos asistentes a las jurisdicciones de la Secretaría de Salud de la Ciudad de México, entre 18 y 83 años, que leyeron y firmaron el consentimiento informado. Se empleó el FCQ-T en la versión española y para el análisis estadístico se ocupó el $R$ Statistical Software, versión 3.4.4.

Se realizó prueba piloto en la Unidad Lázaro Cárdenas del Instituto Politécnico Nacional, con una muestra a conveniencia de 50 sujetos, a quienes se les pidió que formularan observaciones respecto a la redacción. Se realizó análisis de confiabilidad, se adaptó la redacción a regionalismos mexicanos y se pidió a otro grupo que respondiera el cuestionario con modificaciones. Posteriormente se aleatorizaron las jurisdicciones de la Secretaría de Salud de la Ciudad de México de las alcaldías Miguel Hidalgo, Azcapotzalco, Cuauhtémoc y Álvaro Obregón y el cuestionario se aplicó a 1059 adultos sin importar su sexo, asistentes a consulta médica; se les pidió participar voluntariamente y que firmaran el consentimiento informado.

Los análisis estadísticos se llevaron a cabo con $\mathrm{R}$ Statistical Software, versión 3.4.4 (paquete R Psych $^{13}$ y lavaan), ${ }^{14}$ buscando significación a $p<0.05$.

La validez del FCQ-T se verificó mediante análisis factorial exploratorio y confirmatorio. La normalidad de ítems se consideró aceptable para valores asimétricos entre -0.5 y 0.5 y curtosis entre -2 y 2 . Utilizamos el valor de Kaiser-Meyer-Olkin y la prueba de esfericidad de Bartlett para adecuación de los datos para el análisis factorial; valores de Kaiser-Meyer-Olkin < 0.6 indicaron muestreo no adecuado. ${ }^{15}$ Se aplicó análisis factorial exploratorio con rotación oblicua Promax. 
Para crear una propiedad del FCQ-T se extrajeron ítems para cada factor si se cargaron con $\geq 0.35$ en un factor particular, pero $\geq 0.35$ en los otros.

Posteriormente, en el modelo del análisis factorial confirmatorio se utilizó el estimador robusto de máxima verosimilitud, que proporciona errores estándar/índices de ajuste robustos en la naturaleza de los ítems de la escala de Likert y la no normalidad.

Para evaluar que el modelo encajara, se utilizó índice de ajuste comparativo (ICF), el índice Tucker-Lewis (ITL), raíz media estandarizada residual (RMER), y raíz media de error cuadrático de aproximación (RMECA).

Son considerados valores adecuados entre $0.90 \mathrm{y}$ 0.95 para ICF y ITL y entre 0.06-0.08 para RMECA y RMER, mientras valores $>0.95$ para ICF y ITL y los valores $<0.06$ para RMECA y RMER fueron considerados excelentes.

Finalmente, se utilizó el método de regresión para extraer puntuaciones de factores y estadísticos descriptivos de puntuaciones e índices de confiabilidad ( $\alpha$ de Cronbach y coeficientes rho de Dillon-Goldstein) de cada factor que conforman el instrumento. Un valor de coeficiente $>0.80$ indica nivel alto de consistencia interna. La relación entre dominios fue evaluada con correlaciones de Pearson; por encima de 0.81 se consideró excelente, entre 0.61 y 0.80 muy buena y entre 0.41 y 0.60 buena. De los 1059 participantes, $71.86 \%$ (761) fue del sexo femenino; la edad media fue de $39.90 \pm 14.97$.

\section{Resultados}

En el análisis factorial se obtuvo una adecuación simple de 0.977 y con la prueba de esfericidad de Bartlett, $\chi^{2}=31143.93$ y gl $=666$, con $p<0.0001$, lo cual indica adecuación de los ítems para detección de estructura.

Con el análisis Promax, todos los items obtuvieron factores de peso superiores a 0.35 y se reorganizaron de forma distinta a la versión española, por lo que se les cambió el nombre y se redefinieron de la siguiente manera (Tabla 1):

- Factor 1, anticipación y refuerzo por comer: pensar frecuentemente en el alimento que se apetece hace que se recuerden y anticipen las emociones placenteras que se generan tras la ingesta, lo cual reforzará la búsqueda y el consumo del alimento.

- Factor 2, pérdida de control: el intenso deseo por consumir el alimento que genera craving desencadenará la sobreingesta y provocará culpa por ceder al mismo.

- Factor 3, respuesta fisiológica: el craving surge como respuesta fisiológica ante los estímulos físicos o la evocación de recuerdos relacionados con el alimento apetecible y el consumo.

- Factor 4, emociones positivas: el consumo del alimento apetecible se asocia con estados hedónicos que magnificarán la sensación de alivio y bienestar.

Se observó que el FCQ-T explicó $52.91 \%$ de la varianza.

El análisis factorial confirmatorio se realizó utilizando el estimador robusto de máxima verosimilitud. Los estadísticos de bondad de ajuste y los criterios de información del modelo estimado se presentan en la Tabla 2. La prueba de $\chi^{2}$ fue significativa y se analizaron los indicadores de ajuste robusto: RMECA y RMER mostraron excelente bondad de ajuste en el modelo de ecuación estructural y se obtuvo un pobre valor en ICF y ITL.

El análisis factorial confirmatorio reveló carga adecuada de factores estandarizados (anticipación y refuerzo por comer $=0.687-0.869$, falta de control $=0.649-0.802$, respuesta fisiológica $=0.621$ 0.786 , emociones positivas $=0.716-0.802$ ) y todos los intervalos de confianza de carga estandarizada de factor fueron significativos (Tabla 1).

En la Tabla 3 se aprecia la media y desviación estándar de puntuaciones obtenidas de los factores. Se obtuvo un $\alpha$ de Cronbach $=0.973$ y rho de Dillon-Goldstein $=0.975$, lo que indica que es confiable, mientras que los valores obtenidos por factores son $>0.80$. Con el análisis factorial confirmatorio, las correlaciones entre todos los factores fueron excelentes y positivas; todos los intervalos de confianza de coeficientes de correlación fueron estadísticamente significativos.

\section{Discusión}

Se encontró que la fiabilidad del FCQ-T es consistente con investigaciones realizadas en América y Europa $(\alpha \geq 0.90)$. Respecto a la estructura factorial se hallaron diferencias en la cantidad de dimensiones totales, como ha sucedido en diversas validaciones. ${ }^{16-18}$

Estructuralmente se realizó reducción de factores y reagrupamiento de ítems: los 37 que originalmente componían la versión española fueron conservados, ${ }^{19}$ pero reagrupados en cuatro factores consistentes con 
Gaceta Médica de México. 2020;156

Tabla 1. Análisis factorial exploratorio y confirmatorio

\section{Factores e ítems}

\begin{tabular}{|c|c|c|c|c|c|}
\multicolumn{3}{|c|}{ EFA } & \multicolumn{2}{c|}{ AFC } \\
\hline ARE & RF & PC & EP & CEF & IC $95 \%$ \\
\hline
\end{tabular}

Factor 1. Anticipación y refuerzo por comer

17. Cuando como algo que deseo con intensidad me siento culpable (When I eat what I am craving I feel guilty about myself)

20. Siento deseos de comer cuando estoy aburrido(a), enfadado(a), o triste (I crave foods when I feel bored, angry, or sad)

21. Después de comer no tengo tanta ansiedad (I feel less anxious after l eat)

25. No tengo la fuerza de voluntad de resistir mis deseos de comer lo que se me antoja (I have no will power to resist my food crave)

26. Una vez que me pongo a comer algo tengo problemas para dejar de hacerlo (Once I start eating, I have trouble stopping)

27. Por mucho que lo intento, no puedo parar de pensar en comer (I can't stop thinking about eating no matter how hard I try)

28. Gasto demasiado tiempo pensando en lo próximo que voy a comer (I spend a lot of time thinking about whatever it is I will aet next)

29. Si me dejo llevar por la tentación de comer, pierdo todo el control (If I give in to a food craving, all control is lost)

30. A veces me doy cuenta de que estoy soñando despierto y estoy fantaseando con comer (I daydream about food)

31. Cada vez que se antoja una comida sigo pensando en comer hasta que como lo que se me antojó (Whenever I have a food craving, I keep on thinking about eating until I actually eat the food)

32. Cuando tengo muchas ganas de comer algo estoy obsesionado con comer lo que deseo (If I am craving something, thoughts of eating it consume me)

33. A menudo deseo comer cuando siento emociones fuertes (My emotions often make me want to eat)

34. Cada vez que voy a un banquete termino comiendo más de lo que necesito (Whenever I go to a buffet, I end up eating more than what I needed)

35. Para mí es difícil resistir la tentación de tomar comidas apetecibles que están a mi alcance (It is hard for me to resist the temptation to eat appetizing foods that are in my reach)

36. Cuando estoy con alguien que se excede comiendo, yo también me excedo (When I am with someone who is overeating, I usually overeat too)

\begin{tabular}{|c|c|c|c|c|c|}
\hline 0.454 & 0.176 & 0.004 & 0.217 & 0.694 & $0.642-0.747$ \\
\hline 0.587 & 0.054 & 0.116 & 0.077 & 0.725 & $0.681-0.768$ \\
\hline 0.573 & -0.126 & 0.176 & 0.204 & 0.687 & $0.640-0.735$ \\
\hline 0.544 & 0.174 & 0.278 & -0.207 & 0.718 & $0.817-0.876$ \\
\hline 0.767 & 0.168 & -0.025 & -0.027 & 0.847 & $0.817-0.876$ \\
\hline 0.883 & 0.042 & -0.143 & 0.088 & 0.859 & $0.831-0.887$ \\
\hline 0.860 & 0.015 & -0.194 & 0.151 & 0.815 & $0.755-0.854$ \\
\hline 0.937 & 0.065 & -0.175 & 0.022 & 0.869 & $0.840-0.897$ \\
\hline 0.865 & -0.008 & -0.180 & 0.154 & 0.813 & $0.774-0.853$ \\
\hline 0.794 & -0.012 & 0.056 & 0.027 & 0.827 & $0.792-0.862$ \\
\hline 0.799 & -0.008 & 0.024 & 0.037 & 0.820 & $0.783-0.858$ \\
\hline 0.828 & -0.011 & -0.037 & 0.059 & 0.812 & $0.775-0.848$ \\
\hline 0.626 & 0.060 & 0.303 & -0.192 & 0.745 & $0.706-0.784$ \\
\hline 0.621 & 0.138 & 0.283 & -0.205 & 0.779 & $0.743-0.815$ \\
\hline 0.747 & 0.090 & 0.108 & -0.103 & 0.809 & $0.773-0.844$ \\
\hline-0.099 & 0.622 & 0.277 & -0.046 & 0.649 & $0.600-0.697$ \\
\hline 0.151 & 0.758 & -0.029 & -0.071 & 0.782 & $0.764-0.834$ \\
\hline 0.109 & 0.726 & 0.093 & -0.073 & 0.799 & $0.764-0.834$ \\
\hline 0.127 & 0.747 & 0.035 & -0.066 & 0.798 & $0.761-0.835$ \\
\hline 0.028 & 0.736 & 0.001 & 0.102 & 0.778 & $0.739-0.817$ \\
\hline
\end{tabular}

\section{Factor 2. Pérdida de control}

1. Cuando estoy con alguien que está comiendo me da hambre (Being with someone who is eating often makes me hungry)

2. Cuando tengo deseos intensos de comer, una vez que lo hago no puedo parar (When I crave something, I know I won't be able to stop eating once I start)

3. A veces, cuando como lo que se me antoja, pierdo el control y como demasiado (If I eat what I am craving, I often lose control and eat too much)

4. Detesto no poder resistir la tentación de comer (I hate it when I give into cravings)

5. Sin duda alguna, las ganas de comer me hacen pensar en cómo voy a conseguir lo que quiero comer (Food cravings invariably make me think of ways to get what I want to eat) 
Tabla 1. Análisis factorial exploratorio y confirmatorio (Continuación)

\section{Factores e ítems}

\begin{tabular}{|c|c|c|c|c|c|}
\hline \multicolumn{3}{|c|}{ EFA } & \multicolumn{2}{c|}{ AFC } \\
\hline ARE & RF & PC & EP & CEF & IC 95\% \\
\hline
\end{tabular}

Factor 2. Pérdida de control

6. No hago más que pensar en la comida (I feel like I have food on my mind all the $\begin{array}{llllll}-0.045 & 0.675 & 0.138 & 0.112 & 0.753 & 0.712-0.794\end{array}$ time)

7. A menudo me siento culpable cuando deseo ciertas comidas (I often feel guilty for craving certain foods)

8. A veces me encuentro pensativo preocupado con comida (I find myself preoccupied with food)

\section{Factor 3. Respuesta fisiológica}

11. Se me hace agua la boca cuando pienso en mis comidas favoritas (Thinking about my favorite food makes my mouth water)

12. Siento deseos intensos de comer cuando mi estómago está vacío (I crave foods when my stomach is empty)

13. Siento que mi cuerpo me pidiera ciertas comidas (I feel as if my body asks me for certain food)

14. Me da tanta hambre que mi estómago se siente como un pozo sin fondo (I get so hungry that my stomach seems like a bottomless pit)

15. Cuando como lo que deseo, me siento mejor (Eating what I crave makes me feel better)

18. Cada vez que deseo comer algo en particular, me pongo a hacer planes para comer (Whenever I have cravings, I find myself making plans to eat)

19. El comer me tranquiliza (Eating calms me down)

22. Si tengo la comida que deseo, no puedo resistir la tentación de comerla (If I get what I am craving I cannot stop myself from eating it)

23. Cuando se me antoja una comida, normalmente intento comerla tan pronto como pueda (When I crave certain foods, I usually try to eat them as soon as I can)

24. Comer lo que me apetece mucho me sienta estupendamente (When I eat wata I crave I fuel great)

$$
\begin{aligned}
& \begin{array}{llllll}
0.110 & 0.710 & -0.074 & 0.101 & 0.765 & 0.721-0.808
\end{array} \\
& \begin{array}{lllllll}
0.283 & 0.620 & -0.208 & 0.205 & 0.802 & 0.761-0.842
\end{array}
\end{aligned}
$$

\begin{tabular}{|c|c|c|c|c|c|}
\hline-0.226 & 0.322 & 0.635 & 0.085 & 0.621 & $0.578-0.665$ \\
\hline .179 & 0.102 & 0.826 & 0.030 & 0.671 & $0.632-0.710$ \\
\hline
\end{tabular}

\begin{tabular}{|l|l|l|l|l|l}
0.119 & 0.159 & 0.497 & 0.088 & 0.724 & $0.680-0.768$
\end{tabular}

\begin{tabular}{l|l|l|l|l|l}
0.202 & 0.219 & 0.366 & 0.080 & 0.711 & $0.667-0.754$
\end{tabular}

\begin{tabular}{ll|l|l|l|l}
-0.224 & -0.050 & 0.748 & 0.365 & 0.695 & $0.657-0.733$
\end{tabular}

\begin{tabular}{ll|l|l|l|l}
0.301 & 0.059 & 0.353 & 0.174 & 0.734 & $0.695-0.773$
\end{tabular}

\begin{tabular}{lll|l|l|l}
0.208 & -0.216 & 0.357 & 0.578 & 0.734 & $0.695-0.772$
\end{tabular}

\begin{tabular}{ll|l|l|l|l}
0.310 & -0.001 & 0.626 & -0.081 & 0.770 & $0.736-0.803$
\end{tabular}

$\begin{array}{llllll}0.399 & -0.048 & 0.554 & -0.030 & 0.786 & 0.755-0.817\end{array}$

$\begin{array}{llllll}0.099 & -0.170 & 0.719 & 0.192 & 0.740 & 0.706-0.774\end{array}$

\section{Factor 4. Emociones positivas}

9. Como para sentirme mejor (I eat to feel better)

\begin{tabular}{|l|l|l|l|l|l|}
\hline 0.014 & 0.365 & -0.073 & 0.657 & 0.779 & $0.733-0.824$ \\
\hline-0.197 & 0.365 & 0.218 & 0.596 & 0.718 & $0.673-0.764$ \\
\hline 0.343 & 0.029 & 0.133 & 0.460 & 0.802 & $0.768-0.836$ \\
\hline & & & & & \\
\hline 0.228 & -0.192 & 0.207 & 0.638 & 0.716 & $0.672-0.761$ \\
\hline
\end{tabular}

37. Comer me alivia (When I eat food, I feel comforted)

$\mathrm{PC}=$ pérdida de control, $\mathrm{RF}=$ respuesta fisiológica

$\mathrm{AFE}=$ analisis factorial exploratorio, $\mathrm{AFC}=$ analisis factorial contirmatorio, ARE $=$ anticipación y refuerzo por
$E \mathrm{EP}=$ emociones positivas, $\mathrm{CEF}=$ carga estandarizada de factor, IC $95 \%=$ intervalo de confianza a $95 \%$.

Tabla 2. Estadísticos de bondad de ajuste del modelo estimado

\begin{tabular}{|l|c|c|c|c|c|c|}
\hline$x^{2}$ & $g \mid$ & $p$ & ICF & ITL & RMECA & RMER \\
\hline 2588.959 & 623 & 0.0001 & 0.889 & 0.882 & 0.055 & 0.051 \\
\hline
\end{tabular}

$\chi^{2}=$ chi cuadrada del ajuste exato, $g \mathrm{l}=$ grados de libertad, ICF $=$ índice de ajuste comparativo, ITL = índice de Tucker-Lewis, RMECA = error medio cuadrático de aproximación, RMER = raíz media normalizada residual. los restados en otros estudios. ${ }^{16-18} \mathrm{La}$ interpretación de ítems es influida por variables emocionales, comportamentales, cognitivas y contextuales-culturales, lo que podría explicar la rotación de los factores. ${ }^{17}$

Es interesante que en este estudio, el acomodo fue similar al de la validación cubana: ${ }^{18}$ en ambos, en el 
Tabla 3. Estadísticos descriptivos, consistencia interna y correlaciones entre factores

\begin{tabular}{|c|c|c|c|c|c|c|c|c|}
\hline \multirow[t]{2}{*}{ Factores } & \multicolumn{2}{|c|}{$\begin{array}{l}\text { Estadísticos } \\
\text { descriptivos }\end{array}$} & \multicolumn{2}{|c|}{ Consistencia interna } & \multicolumn{4}{|c|}{ Correlaciones } \\
\hline & Media & DE & $\begin{array}{c}\alpha \text { de } \\
\text { Cronbach }\end{array}$ & $\begin{array}{l}\text { Rho de Dillon- } \\
\text { Goldstein }\end{array}$ & ARE & PC & RF & EP \\
\hline Anticipación y refuerzo por comer & 0.000 & 1.155 & 0.960 & 0.965 & & $0.859^{*}$ & $0.854^{*}$ & $0.865^{\star}$ \\
\hline Pérdida de control & 0.000 & 1.115 & 0.920 & 0.935 & $0.842-0.874^{* *}$ & & $0.819^{*}$ & $0.852^{*}$ \\
\hline Respuesta fisiológica & 0.000 & 1.201 & 0.913 & 0.929 & $0.837-0.870^{\star *}$ & $0.798-0.838^{\star \star}$ & & $0.936^{*}$ \\
\hline Emociones positivas & 0.000 & 1.169 & 0.838 & 0.894 & $0.849-0.879^{* *}$ & $0.835-0.868^{* *}$ & $0.928-0.943^{\star *}$ & \\
\hline
\end{tabular}

$\mathrm{DE}=$ desviación estándar; $\mathrm{ARE}=$ anticipación y refuerzo por comer; $\mathrm{PC}=$ pérdida de control; $\mathrm{RF}=$ respuesta fisiológica; $\mathrm{EP}=$ emociones positivas. ${ }^{*}$ Correlación de Pearson. ${ }^{\star *}$ Intervalo de confianza a $95 \%$

factor 1 se observan los elementos que se refieren a los refuerzos positivos que genera la ingesta.

Los datos obtenidos en esta investigación muestran que hay pensamientos relacionados con alimentos que provocan emociones placenteras, lo que motiva la sobreingesta. La diferencia radica en los ítems: 17 ("cuando como algo que deseo con intensidad me siento culpable"), 20 ("siento deseos de comer cuando estoy aburrido[a], enfadado[a], o triste"), 21 ("después de comer no tengo tanta ansiedad") y 22 ("si tengo la comida que deseo, no puedo resistir la tentación de comerla") se agrupan diferente en la versión cubana ${ }^{18}$ y en esta versión se han acomodado en el primer factor, que explica que el consumo desencadena culpa, aburrimiento, enojo o tristeza por no poder resistir a la tentación.

El factor 2 fue nombrado pérdida de control, consistente con la versión holandesa ${ }^{16}$ y cubana. ${ }^{18}$ En la primera, los ítems 2 ("cuando tengo deseos intensos de comer, una vez que lo hago no puedo parar") y 3 ("a veces, cuando como lo que se me antoja, pierdo el control y como demasiado") coincidieron. ${ }^{16}$ Mientras que en la cubana, los ítems 2, 3 y 1 ("cuando estoy con alguien que está comiendo me da hambre") ${ }^{18}$ coinciden. Los otros ítems integrados a este factor explican la intensidad del deseo por comer cierto tipo de alimentos que causan sobreingesta y sentimientos de culpa. A diferencia de lo observado por Rodríguez Martín y Molerio Pérez, ${ }^{18}$ la pérdida de control no se debe a señales ambientales y sí a características intrínsecas del sujeto que lo motivan a consumir impulsivamente. ${ }^{19}$

El factor 3 se denominó "respuesta fisiológica" a partir de la respuesta condicionada al hambre, como salivación al recordar comida, sensación de "no saciedad", "estar insatisfecho" y sensación de placer al comer. Los ítems 11 ("se me hace agua la boca cuando pienso en mis comidas favoritas"), 13 ("siento que mi cuerpo me pide ciertas comidas") y 14 ("me da tanta hambre que mi estómago se siente como un pozo sin fondo") coinciden con el factor 5 (craving como un estado fisiológico) de la versión original, ${ }^{11}$ la española ${ }^{20}$ y brasileña ${ }^{21}$ y con el factor 6 (renombrado como "hambre") en la versión alemana. ${ }^{17}$

El último factor (factor 4) se denominó "emociones positivas". A diferencia de lo que proponen Rodríguez Martín y Molerio Pérez, ${ }^{18}$ que explican este factor como "comer para regular emociones o ingesta emocional". Sin embargo, ambos factores solo coinciden en los ítems 19 ("el comer me tranquiliza") y 37 ("comer me alivia"). En la presente investigación se sumaron los ítems mencionados y otros fueron reagrupados (Tabla 1). El consumo está asociado con emociones positivas, por lo que la sensación de recompensa se magnifica.

\section{Agradecimientos}

A la Secretaría de Salud de la Ciudad de México, en especial al licenciado Leopoldo Galván Pérez, responsable de Medicina a Distancia.

\section{Bibliografía}

1. Al-Nakeeb $Y$, Lyons M, Dodd LJ, Al-Nuaim A. An investigation into the lifestyle, health habits and risk factors of young adults. Int J Environ Res Public Health. 2015;12:4380-4394.

2. El-Kassas G, Ziade F. Exploration of the dietary and lifestyle behaviors and weight status and their self-perceptions among health sciences university students in North Lebanon. BioMed Rest Int. 2016;2016:9762396.

3. Berridge KC, Ho CY, Richard JM, DiFeliceantonio AG. The tempted brain eats: pleasure and desire circuits in obesity and eating disorders. Brain Res. 2010;1350:43-64.

4. Frankort A, Roefs A, Siep N, Roebroeck A, Havermans R, Jansen A. The craving stops before you feel it: neural correlates of chocolate craving during cue exposure with response prevention. Cereb Cortex. 2014; 24:1589-1600.

5. Mühlberg C, Mathar D, Villringer A, Horstmann A, Neumann J. Stopping at the sight of food: how the gender and obesity impact on response inhibition. Appetite. 2016;107:663-676.

6. Iani L, Barbaranelli C, Lombardo C. Cross-validation of the reduced form of the Food Craving Questionnaire-Trait using confirmatory factor analysis. Front Psychol. 2015;6:433. 
7. Pelchat ML. Of human bondage: Food craving, obsession, compulsion, and addiction. Physiol Behav. 2002;76:347-352.

8. Tiggemann M, Kemps $\mathrm{E}$. The phenomenology of food cravings: the role of mental imagery. Appetite. 2005;45:305-313.

9. Pelchat ML, Johnson A, Chan R, Valdez J, Ragland JD. Images of desire: food-craving activation during fMRI. Neuroimage. 2004;23: 1486-1493.

10. Potenza MN, Grilo CM. How relevant is food craving to obesity and its treatment? Front Psychiatry. 2014:5:164.

11. Cepeda-Benito A, Gleaves DH, Williams TL, Erath SA. The development and validation of the state and trait food-cravings questionnaires. Behav Res Ther. 2000;31:151-173.

12. Avitia GC, Loya-Méndez Y, Portillo-Reyes V, Reyes-Leal G, Capps Iv JW Cravings, sugar and fat consumption as determinant factors of obesity in young adults in Juarez City. Nutr Hosp. 2018:35:833-840.

13. psych. Procedures for personality and psychological research [En línea] EE. UU.: Northwestern University; 2017.

14. Rosseel $Y$. lavaan: an $R$ package for structural equation modeling. J Stat Software. 2012:48:1-36.

15. Kaiser HF. An index of factorial simplicity. Psychometrika. 1974;39:31-36.
16. Nijs I, Franken I, Muris P. The modified Trait and State Food-Cravings Questionnaires: development and validation of a general index of food craving. Appetite. 2007;49:38-46.

17. Meule A, Lutz A, Vögele $C$, Küber A. Food cravings discriminate differentially between successful and unsuccessful dieters and non-dieters. Validation of the Food Cravings Questionnaires in German. Appetite. 2012;58:88-97.

18. Rodríguez-Martín, BC, Molerio-Pérez O. Exploring the factor structure of the Food Cravings Questionnaire-Trait in Cuban adults. Front Psychology. 2014;5:1-12

19. Veilleux JC, Hill MA, Skinner KD, Pollert GA, Baker DE, Spero KD. The dynamics of persisting through distress: development of a momentary distress intolerance scale using ecological momentary assessment. Paschoal Asses. 2018;30:1468-1478.

20. Cepeda-Benito A, Gleaves DH, Fernández MC, Vila J, Williams TL, Reynoso J. The development and validation of Spanish versions of the state and trait food cravings questionnaires. Behav Res The. 2000;38:1125-1138.

21. Queiroz-De Medeiros AC, Campos-Pedrosa LF, Hutz CS, Yamamoto ME. Brazilian version of food cravings questionnaires: psychometric properties and sex differences. Appetite. 2016;105:328-333. 